\title{
The value of portable renal hilar Doppler ultrasound screening (RHDUS) for renal artery stenosis in critically-ill patients
}

\author{
Jacob Hayes, Mohammad Amarneh*, Janet Pollard, Steven Morales and Monzer Abu-Yousef \\ Department of Radiology, University of Iowa Hospitals and Clinics, Iowa City, USA
}

\begin{abstract}
Purpose: To evaluate the efficacy and utility of portable Renal Hilar Doppler Ultrasound (RHDUS) screening for Renal Artery Stenosis (RAS) in critically-ill patients.

Materials and methods: A retrospective study of adult Intensive Care Unit (ICU) patients receiving RHDUS to rule out RAS spanning a time frame of one year. Patients receiving RHDUS were included with the exclusion of patients under 18 years, and exams of poor diagnostic quality. Patients' sex, age, serum creatinine and BUN, risk factors for RAS, and primary and secondary indications for the requested Doppler exam were included. Renal Doppler results and confirmatory studies such as Angiography, MRA, CTA, etc. were included when available.
\end{abstract}

Results: 291 (90\%) of 325 Doppler studies were included in the study. Twenty-one of the studies met criteria for possible RAS. Sensitivity and specificity was $80 \%$ and 98.5\% respectively. A significant association between hypertension-related indications and RAS-positive studies was shown ( $\mathrm{p}=.002)$.

Conclusion: RHDUS is a useful screening test in patients with a high suspicion of RAS. In many ICU patients presenting only with AKI or hemodynamic instability, the use of RHDUS screening for RAS is very low yield and should probably not considered an appropriate test.

\section{Introduction}

Renal artery stenosis (RAS) is a common renovascular disease defined by the narrowing of the renal arteries leading to reduced perfusion. This renal ischemia can lead to the overproduction of renin and ultimately renal hypertension. Though not all cases of RAS are clinically significant and its prevalence in the general population is not known, it is considered one the most common causes of secondary hypertension and is estimated to be the main contributor in up to $5 \%$ of all hypertensive cases [1,2]. Up to $90 \%$ of RAS is caused by atherosclerotic plaques, occurring primarily in the elderly population, and the majority of the remaining cases are due to fibromuscular dysplasia most commonly seen in females under 40. Other rare causes of RAS include vasculitis, thromboembolism, aortic dissection, and renal artery aneurysms. RAS most commonly presents as resistant or uncontrollable hypertension, renal failure secondary to ischemic nephropathy, recurring episodes of congestive heart failure, or flash pulmonary edema $[3,4]$.

Conventional renal Doppler ultrasonography (RDUS) performed in ambulatory patients is often used for the detection of renal artery stenosis due to its noninvasive nature and relatively low cost. It has also been shown by many studies to have high sensitivity and specificity when compared to renal angiography, the gold standard for RAS detection [1,5-10]. This exam includes direct visualization of the renal artery origin and evaluation of the renal poles for accessory and main artery branch stenosis.

In the intensive care units where kidney injury can occur as often as $50 \%$ of the time [11], patients commonly undergo screening for
RAS by Doppler ultrasound. Because of their non-ambulatory state, these critically-ill patients receive a portable and suboptimal form of the Doppler exam, limiting views to the hilar artery only the Doppler evaluation of indirect parameters of acceleration time and index. In this hilar Doppler study (RHDUS) there is no direct assessment of the renal artery origin or accessory arteries. A 2001 study [12-14] looking at using these indirect Doppler parameters as a screening tool for diagnosing RAS found that in a pre-selected group of hypertensive patients, indirect parameters were a useful screening tool. However these indirect parameters have not been studied in the critically-ill population in which they are being employed (Figures 1a and $1 \mathrm{~b}$ ).

Even though RDUS is a studied modality of RAS screening and is used in studies looking at the prevalence of RAS [13]. The more limited RHDUS has not been compared to RDUS in its efficacy as a screening tool. However, RHDUS has been shown to have high accuracy when compared to arteriography using the Acceleration Time (AT) and Acceleration Index (AI) [15] (Figure 1c), and is most useful in the setting of a high pre-test probability of RAS and for surveillance following revascularization. But the modality is limited by the presence of multiple arteries, occlusion, and a high incidence of false positives in postoperative and hemodynamically unstable

Correspondence to: Mohammad Amarneh, MBBS, Department of Radiology, University of Iowa Hospitals and Clinics, Iowa City, IA 52242, USA, Tel: 337852-8528, Email: mohammad-amarneh@uiowa.edu

Received: December 22, 2016; Accepted: January 05, 2017; Published: January 09,2017 


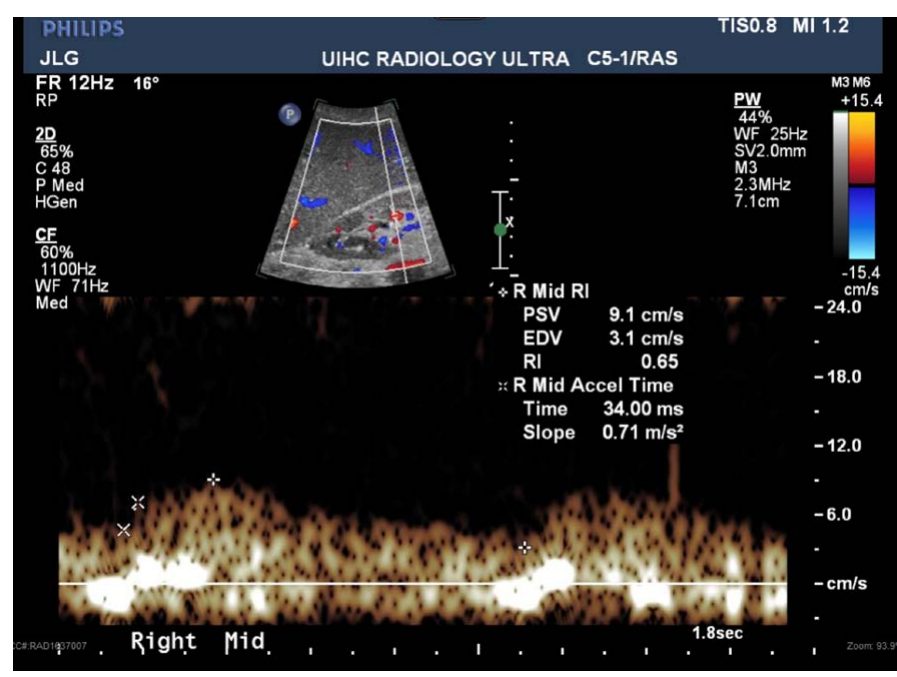

Figure 1a. 63 year old male with acute kidney injury and uncontrolled hypertension. RDHUS shows classic slow rising systolic peak, so called "Tardus parvus", with decreased acceleration index or slope. Findings are consistent with renal artery stenosis.

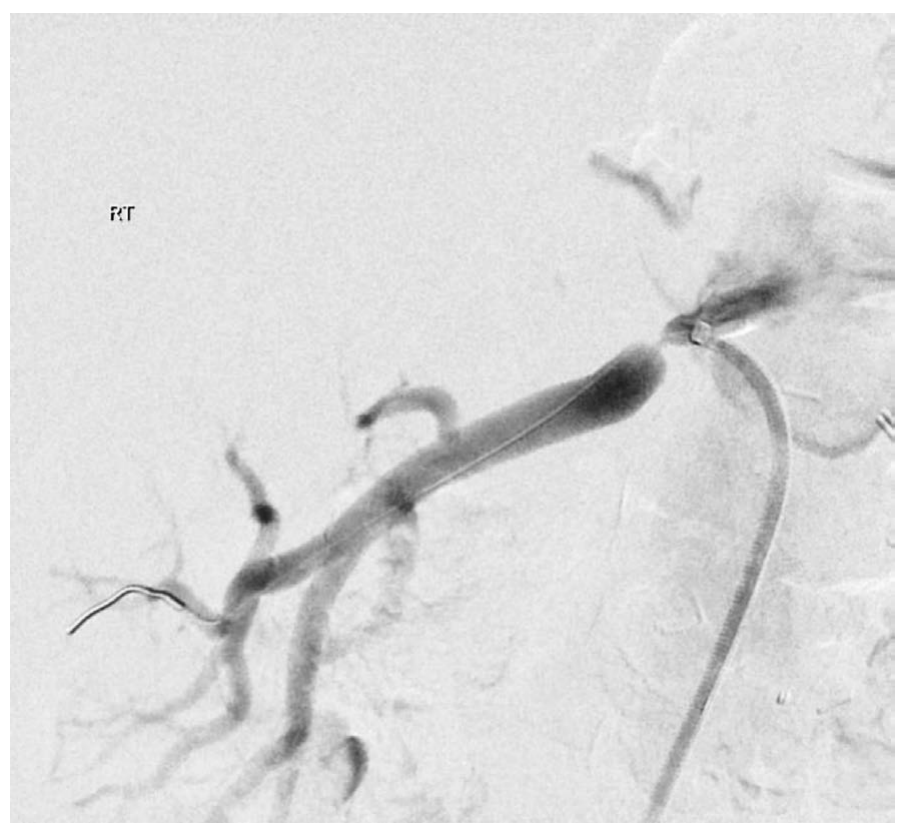

Figure 1b. Digital Subtracted Angiogram of the right renal artery shows severe focal stenosis at the proximal right renal artery. This was treated with stent, and post stent angiogram showed no significant residual stenosis (not shown).

patients. Doppler generally also does not detect stenosis in mild to moderate disease $(<60 \%)$ leading to high false negative rates [15]. Given the hemodynamic instability of many critically-ill patients, we will explore the question of whether RHDUS is an effective screening tool when ruling out RAS in the ICU. We have hypothesized the rate of management-altering positive studies to be less than $5 \%$.

\section{Materials and methods}

Reports of all portable RHDUS examinations performed in adult critical care units (i.e., medical, surgical, and cardiovascular) were reviewed retrospectively for the interval of January 1, 2011 to December 31, 2012. Also reviewed was the electronic medical record of those patients. All ultrasounds were performed portably with the acceleration time and index taken from the kidney hilum. The Doppler

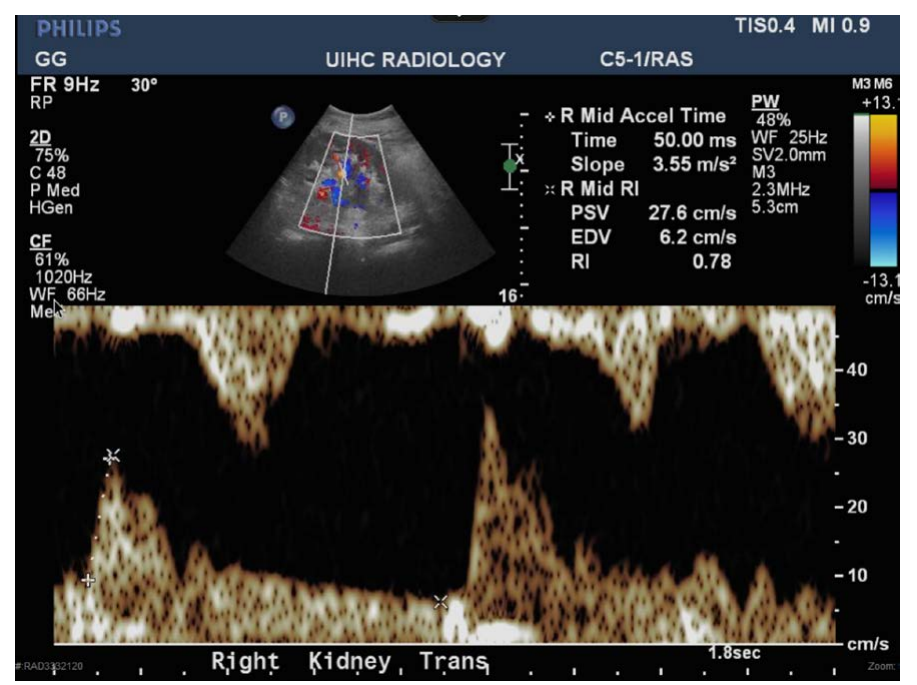

Figure 1c. Repeat RHDUS showssignificantly improved renal arterial flow, with normal early systolic peak, and normal acceleration index.

angle was always targeted for 0 degrees; in cases where the angle was not 0 degrees, the angle was corrected for. All exams were performed by certified sonographer technicians on Acuson Sequoia 512 and Philips IU22 machines. Images were read and interpreted by a board certified radiologist with or without the assistance of a radiology resident. Our Institutional Review Board approved the study.

Exclusion criteria included patients under the age of 18 and exams of non-diagnostic quality. Non-diagnostic quality images resulted from issues such as beam attenuation from body habitus or bowel gases, patient position or un-cooperation, inability to hold breath during the exam, wounds or bandages over areas that were required for optimal imaging, irregular Doppler flow caused by a present LVAD or intraaortic balloon pump, or other patient-specific issues making Doppler results unobtainable or unreliable. Patients with both kidneys, but had a Doppler scan with unilateral diagnostic quality were also excluded. This is because renal artery stenosis occurs unilaterally in most patients $[12,13]$ and these exams were not adequate to define the presence or absence of RAS in the patient based on a unilateral renal scan. From the medical record was recorded the following: sex; age; serum creatinine $(\mathrm{mg} / \mathrm{dL})$ and blood urea nitrogen concentrations $(\mathrm{mg} / \mathrm{dL})$; number of kidneys; principal diagnosis for admission to ICU; documented history of hypertension, chronic kidney disease, diabetes mellitus, and/or cardiovascular disease; and clinical indications for Doppler screening such as acute kidney injury, hypertensive urgency, flash pulmonary edema, aortic dissection, and sepsis. The criterion for acute or acuteon-chronic kidney injury was defined as per the patient medical record. BUN to serum creatinine ratio was also calculated.

From the radiology report was recorded the clinical indication for the exam, the presence or absence of renal artery stenosis as reported by the interpreting radiologist based on acceleration time and indices, resistive indices, early systolic peaks, and pertinent grayscale sonographic findings such as kidney length and echogenicity. Criteria for $60 \%$ stenosis by indirect parameters included acceleration index of $<300 \mathrm{~cm} / \mathrm{s}^{2}$, acceleration time of $>70 \mathrm{msec}$, diminished or loss of early systolic peaks and abnormal waveform shape, or resistive index of $<0.55$. A resistive index of $>0.7$ was suggestive of microvascular or parenchymal disease. Peak systolic velocity measurements were not collected because, though occasionally available, they are not routinely 
evaluated as part of the portable exam. Unobtainable Doppler signal and waveforms due to arterial occlusion were considered abnormal findings if not explained by technical issues. Studies that were normal in all measurements except for the loss of early systolic peaks were not considered as positive findings for significant renal artery stenosis. Studies have shown that loss of early systolic peak alone is not a good indicator of renal artery stenosis [14]. Also noted was the presence or absence of microvascular/medical renal disease by Doppler evidence, renal vein thrombosis, hydronephrosis, stones, and focal lesions excluding simple cysts. Confirmatory tests for RAS-positive Doppler studies were also recorded. These included renal angiography, MRA, and CTA. Also recorded was the patients' kidney function at discharge from intensive care (Table 1).

Dichotomous variables were analyzed by $\chi^{2}$ tests and continuous variables were analyzed with $t$ tests. Sensitivity and specificity were calculated by comparison with angiography, MRA, CTA, or other confirmatory testing available.

\section{Results}

A total of 318 patients received 325 renal Doppler exams from January 2011 through 2012. Out of all exams, 89.5\% (291) Doppler exams were technically successful and included in the analysis. The remaining exams were not technically adequate to predict the presence

Table 1. Summary of statistics on patients examined by portable renal Doppler ultrasound from January 1, 2011 to December 31, 2012 in hospital ICUs.

\begin{tabular}{|c|c|}
\hline Demographics and PMH & Successful Exams $(n=291)$ \\
\hline Age in years (mean, SD) & $60.3 \pm 15.7$ \\
\hline \multicolumn{2}{|l|}{ Gender } \\
\hline -Male & $164(56.4 \%)$ \\
\hline -Female & $127(43.6 \%)$ \\
\hline Hypertension & $213(73.2 \%)$ \\
\hline Chronic kidney disease & $88(30.2 \%)$ \\
\hline Diabetes mellitus/hyperglycemia & $106(36.4 \%)$ \\
\hline Cardiovascular disease (CAD, PVD, or CHF) & $123(42.3 \%)$ \\
\hline -Coronary artery disease & $87(29.9 \%)$ \\
\hline -Peripheral vascular disease & $29(10.0 \%)$ \\
\hline -Congestive heart failure & $72(24.7 \%)$ \\
\hline Acute or acute-on-chronic kidney injury & $244(83.8 \%)$ \\
\hline Serum creatinine (mean, SD) & $3.6 \pm 3.6$ \\
\hline Blood Urea Nitrogen (mean, SD) & $53.6 \pm 38.3$ \\
\hline BUN to creatinine ratio (mean, SD) & $18.4 \pm 10.7$ \\
\hline Hypertensive urgency/flash pulmonary edema & $72(24.7 \%)$ \\
\hline Hypertension in setting of AKI & $35(12.0 \%)$ \\
\hline Hypertension without AKI & $37(12.7 \%)$ \\
\hline $\begin{array}{l}\text { Other: } \mathrm{CHF}, \mathrm{CKD}, \mathrm{AAAR} \text {, hematuria, renal atrophy, } \\
\text { aortic dissection, urosepsis/shock, abdominal bruit }\end{array}$ & $10(3.4 \%)$ \\
\hline Renal artery stenosis/occlusion & $21(7.2 \%)$ \\
\hline -True Positive & $8(2.7 \%)$ \\
\hline -False Positives & $4(1.4 \%)$ \\
\hline -Unknown (unconfirmed) & $9(3.1 \%)$ \\
\hline RAS known false negatives & $2(0.7 \%)$ \\
\hline Microvascular/medical renal disease & $256(88.0 \%)$ \\
\hline Renal vein thrombosis & $3(1.0 \%)$ \\
\hline Hydronephrosis & $17(5.8 \%)$ \\
\hline Renal calculi & $7(2.4 \%)$ \\
\hline $\begin{array}{l}\text { Focal lesions } \\
\text { (excluding simple renal cysts) }\end{array}$ & $11(3.8 \%)$ \\
\hline Resistive Index (mean, SD) & $0.81 \pm 0.09$ \\
\hline Kidney length (mean, SD) & $11.6 \pm 1.7$ \\
\hline
\end{tabular}

or absence of renal artery stenosis and were excluded. This was caused by extremely poor diagnostic quality due to technical issues or patient un-cooperativity. Of the 34 technically inadequate exams, 7 were completely non-diagnostic and 27 exams were incomplete. It should be noted that of these exams excluded from further review, none yielded results, however minimal, that were suggestive of renal artery stenosis. Included in the analysis were 25 exams that were reported as suboptimal due to previously mentioned technical reasons but contained complete data for both kidneys. It should be noted that all exams analyzed in this study were regarded as suboptimal on the basis that they were performed by portable technique and limited to the evaluation of the hilar artery and lacked the ability to rule out accessory renal artery stenosis or minimal to moderate disease.

All patients were reviewed regardless of clinical indication for the renal Doppler screening. Though not all patients were RAS suspects, the presence or absence of RAS is evaluated in each examination. Table 1 is a statistical summary of all demographic data and Doppler findings of the 291 examinations performed. The primary indication for RHDUS evaluation was acute or acute on chronic renal failure (84\%). One quarter of the RHDUS studies were clinically indicated by uncontrolled hypertension, hypertensive urgency, or flash or pulmonary edema with or without concurrent acute kidney injury. Less than $1 \%$ of the patients (10 cases) had neither acute kidney injury nor resistant/uncontrolled hypertension. The primary indication for RHDUS in these patients was congestive heart failure, chronic kidney failure, aortic dissection, urosepsis/UTI, presence of abdominal bruits on exam, history of renal infarct, hematuria, and renal atrophy. Of these 10 cases, five had a documented history of hypertension, one had previously known renal artery stenosis with stent placement, and one received renal artery reimplantation post aortic aneurysm rupture. Other clinical indications for Doppler exams in patients with concurrent acute kidney injury or hypertensive urgency included: peritonitis, endocarditis, hepatitis/ cirrhosis, anasarca, anemia, thromboembolic disease, organ transplant, chemical burns, hemorrhage, vasculopathies, hypotension, obstructive uropathy, arterial occlusion, myocardial infarction, systemic lupus erythematosus crisis, heart valve replacement, and nephrectomy.

Ages for examined patients ranged from 20.2 to 95.9 years (mean, 60.3; median, 61.3). 164 exams were performed on males (56\%) and 127 exams were for females (44\%), (Table 2). Seven patients had solitary kidneys. Seven patients had previously documented renal artery stenosis and 2 had angioplasty with stent placement. Serum creatinine levels ranged from 0.4 to $33.6 \mathrm{mg} / \mathrm{dL}$ (mean, 3.6; median, 2.6). BUN ranged from 4 to $217 \mathrm{mg} / \mathrm{dL}$ (mean, 53.6; median, 43). BUN to serum creatinine ratio ranged from 3 to 82.5 (mean, 18.4; median 15.8). 99 exams (34\%) were performed on patients with BUN to creatinine ratio

Table 2. Prevalence of Doppler findings indicative of treatable RAS by age and sex.

\begin{tabular}{|c|c|c|}
\hline \multicolumn{2}{|c|}{ Category (no. of exams) } & RAS indicated \\
\hline \multicolumn{2}{|c|}{ Overall $(n=291)$} & $7.2 \%$ \\
\hline \multicolumn{3}{|l|}{ Sex } \\
\hline & Male $(n=164)$ & $8.5 \%$ \\
\hline & Female $(\mathrm{n}=127)$ & $5.5 \%$ \\
\hline \multicolumn{3}{|c|}{ Age* } \\
\hline & $<50(\mathrm{n}=77)$ & $3.9 \%$ \\
\hline & $50-59(n=61)$ & $8.2 \%$ \\
\hline & $60-69(n=69)$ & $10.1 \%$ \\
\hline & $70-79(\mathrm{n}=58)$ & $5.2 \%$ \\
\hline & $>80(\mathrm{n}=26)$ & $11.5 \%$ \\
\hline * at & of exam & \\
\hline
\end{tabular}


of 20 or greater. 213 (73\%) examined patients had a documented history of hypertension. 88 (30\%) had documented chronic kidney disease. 106 (36\%) had documented diabetes mellitus or hyperglycemia. $123(42 \%)$ had documented cardiovascular disease (i.e., coronary artery disease, peripheral vascular disease, or congestive heart failure).

Doppler findings included 21 exams (7\%) suggestive of RAS. Other positive findings from RHDUS included: microvascular renal disease $(88 \%)$, possible renal vein thrombosis $(1 \%)$, hydronephrosis (6\%), renal calculi (2\%), and focal lesions excluding simple cysts (4\%). Kidney length ranged from $4.5 \mathrm{~cm}$ to $19.2 \mathrm{~cm}$ (mean, 11.6; median 11.6). Resistive indices ranged from 0.5 to 1.0 (mean 0.8 ; median 0.8 ). Table II shows the prevalence of RAS-positive studies compared by age strata and sex. A summary of the patients with a RAS-positive RHDUS can be found in Table 3 .

RAS: Of 291 exams, 21 (7\%) had abnormal findings near or outside the indirect parameters for RHDUS. This suggested either a diagnosis of true renal artery stenosis or the inability to rule it out by Doppler alone; 4 of the 21 exams were positive by unobtainable waveforms and possible occlusion. Of the 7 patients with a history of renal artery stenosis, 4 had RAS-positive scans.

Confirmatory testing was available in 12 of the 21 RAS-positive studies. 8 out of the 12 were confirmed with follow-up imaging. Renal angiography confirmed 3 cases, while 2 were confirmed with abdominal CT and one by conventional RDUS in an ultrasound suite. A single study showed renal atrophy and no flow on a nuclear flow and function study that was confirmatory of renal artery stenosis/occlusion. An occlusive thrombus was found in one patient by aortic angiogram post repair of a ruptured thoracic aortic aneurysm. Outcomes for the 8 confirmed RAS patients included stent placement, angioplasty, hemodialysis, endarterectomy, and no intervention with discharge.

Of the remaining RAS-positive studies with follow-up, 3 were ruled out by CTA, angiogram, and RDUS. One study that met RAS criteria was considered a false positive because the symptoms fit closer with congestive heart failure and went on to receive an LVAD.

The remaining 9 abnormal exams did not receive any type of secondary studies to confirm or rule out RAS. MRA was scheduled for one patient that was not completed due to her mental status. In another 4 study reports, it was recommended by radiology that confirmatory testing be done but was not completed. The remainder had no record of confirmatory testing or a diagnosis of RAS recorded in the medical record even though the radiologist reported findings consistent with RAS based on the Doppler study. One patient with severe hydronephrosis received a nuclear flow and function study that showed high grade obstruction but was not capable of commenting on the possibility of RAS. Outcomes for these patients included 7 with improved of stable renal function upon discharge, one patient was discharged to palliative care and the other was discharged on dialysis. Table 3 shows a summary of all patients with RAS-positive findings on Doppler and their outcome.

Three patients under the age of 50 were RAS-positive on RHDUS. The youngest patient was 39 . All 3 had a history of hypertension while 2 had poorly controlled hypertension and congestive heart failure. One of these 2 had RAS confirmed by angiography and was treated with stent placement. The other patient under 50 had AKI and had a history of diabetes. Neither remaining patient had confirmatory studies completed, and the significance of their positive RHDUS is unknown.

Table 3. Patients with positive results for RAS. Summary of ICU stay and outcomes based on RAS diagnosis.

\begin{tabular}{|c|c|c|}
\hline Admission/Indication for RHDUS & Findings on RHDUS and follow-up studies & Outcome of kidney function \\
\hline \multicolumn{3}{|l|}{ True Positives* (8) } \\
\hline HTN emergency & Unilateral; confirmed on angiography & Angioplasty and stent \\
\hline Ischemic cardiomyopathy/AKI & Unilateral; no flow, atrophy; $3 \%$ function on Nuclear flow \& function & Non-functioning; Hemodialysis \\
\hline Ischemic bowel/AKI \& RAS & Unilateral; atrophy on CT, possible occlusion & Improved \\
\hline TAAR/renal artery reimplant & Occlusion; confirmed occlusive thrombus on angiogram & Endarterectomy; stable function \\
\hline HTN emergency/RAS & Bilateral; confirmed by angiography & Bilateral angioplasty \\
\hline Pneumonia/AKI \& RAS & Occlusion; near occlusion seen on previous Doppler & Improved \\
\hline STEMI/CKD \& RAS & Near occlusion; seen previously on angiogram and Doppler & Improved \\
\hline Stroke/Renal atrophy & $\begin{array}{l}\text { Unilateral, not visualized; abdominal CT renal artery origin } \\
\text { calcifications and atrophy }\end{array}$ & End-of-life care \\
\hline \multicolumn{3}{|l|}{ False Positives $\dagger(4)$} \\
\hline HTN emergency & Unilateral; CTA showed single artery with no stenosis & Improved \\
\hline $\mathrm{AKI} / \mathrm{HTN}$ & Bilateral; ruled out by full Doppler & No evidence of RAS; Improved \\
\hline HTN emergency & Bilateral; ruled out by angiogram & Normal renal arteries; Improved \\
\hline CHF & Bilateral; more likely due to $\mathrm{CHF}$ & LVAD placement; Improved \\
\hline \multicolumn{3}{|l|}{ Unknown Significance $\$$ (9) } \\
\hline AKI & Unilateral; second study recommended & Improved \\
\hline $\mathrm{CHF} / \mathrm{HTN}$ & Unilateral; second study recommended & Improved \\
\hline Herniated Disk/AKI & Unilateral; second study recommended if indicated & improved \\
\hline Renal Failure/HTN & Unilateral & Hemodialysis \\
\hline Sepsis/AKI & Bilateral; second study recommended & Improved \\
\hline Hypoxia/AKI & Unilateral; atrophy w no waveform, possible occlusion & Improved; transferred to VA \\
\hline GI bleed/AKI & Unilateral; second study recommended & Improved \\
\hline Stroke/AKI & Unilateral w severe hydronephrosis; Nuclear flow \& Function cannot rule out arterial stenosis & $\begin{array}{l}\text { Severe hydronephrosis with high- } \\
\text { grade obstruction; Improved }\end{array}$ \\
\hline MCA infarct/HTN & Bilateral; MRA not completed & End-of-life care; deceased \\
\hline \multicolumn{3}{|c|}{$\begin{array}{l}\text { *Intervention or diagnosis of RAS found in medical record } \\
\text { †Diagnosis of RAS unlikely due to secondary study or Radiologist's impression of Doppler results } \\
\text { †Unknown significance of Doppler results due to lack of secondary study or diagnosis in the medical record }\end{array}$} \\
\hline
\end{tabular}


The oldest patient with RAS findings was 96 years old. All but one patient with a RAS-positive study over the age of 50 had documented hypertension and the single patient without documented hypertension had no documented medical history available at the time of admission to the ICU.

269 exams were negative for RAS. 3 exams were normal except for the loss of early systolic peaks. Of the 7 patients with a known history of RAS, 3 had negative findings on Doppler; one of these patients had previously placed renal artery stents.

Fourteen patients had unilateral evidence of renal artery stenosis and 7 were confirmed with secondary studies. Only one was ruled out by CTA. 6 were not followed up or confirmed. Bilateral RAS was seen in 7 studies and 3 were ruled out by secondary testing. Angioplasty confirmed one case of bilateral stenosis and 3 cases were not confirmed or followed up.

Univariate analysis revealed no significant differences between patients with positive RAS findings and negative RAS findings in age, gender, BUN to creatinine ratio, kidney length, or history of diabetes, cardiovascular, atherosclerotic disease, or acute or chronic renal insufficiency. All but one patient with positive RAS findings (96\%) had a documented history of hypertension compared to $71 \%$ among those negative RAS findings ( $\mathrm{p}=.014)$. Half of the RAS-positive cases were in intensive care with uncontrolled hypertension or hypertensive emergency/flash pulmonary edema compared to $23 \%$ among RASnegative patients $(\mathrm{p}=.004)$. Though there is significant difference in resistive index, RAS-positive RIs were still elevated above the normal range with a mean of 0.74 . Table IV is a summary of prevalence of variables in RHDUS RAS-positive studies and RAS-negative exams (Table 4).

MRD: Microvascular or medical renal disease (MRD) was seen in $88 \%$ of our patients. Univariate analysis showed a significant association between MRD-positive study and age $(\mathrm{p}=.004)$, diabetes mellitus $(\mathrm{p}=.004)$, and the clinical indication of acute kidney injury $(\mathrm{p}=.033)$.

\section{Discussion}

The technical success of portable renal hilar Doppler US in this study was $90 \%$. This is comparable to success in other studies of renal Doppler ranging from $86 \%$ to $94 \%[5,7,15]$. Of all RHDUS studies analyzed, $7 \%$ showed evidence of RAS. The incidence of RAS in the population of patients over 65 years old was reported by Hansen, et al. [13] to be near $7 \%$ in the general population. However, other studies have shown the prevalence to much higher-as much as $40 \%$ in select populations with or at high risk for atherosclerotic disease $[1,12,16]$. In our population of critically-ill patients, there was a prevalence of nearly $10 \%$ among those over 65 and $8 \%$ among those with documented cardiovascular or atherosclerotic disease. The lower than expected prevalence of RAS among patients with known atherosclerosis may represent the limitations of RHDUS to detect mild to moderate stenosis. This could call into question the efficacy of RHDUS in screening for RAS; however, mild to moderate disease is typically asymptomatic and intervention is not performed on stenosis less than $60 \%$. Screening for RAS is for symptomatic disease where intervention is likely to benefit the patient.

Conventional RDUS is currently used as a screening tool for RAS because of the low cost and non-invasive nature. High sensitivities and specificities measured using peak systolic velocity (PSV) and the renal to aortic ratio (RAR) reported between $84 \%$ to $98 \%$ and $62 \%$ to $90 \%$ respectively and positive and negative predictive values between $94 \%$ and $99 \%$ by multiple studies $[1,5,6,8,9]$. RHDUS measuring indirect parameters such as acceleration index (AI) and time (AT) has also been shown in studies to have comparable sensitivities and specificities $89 \%$ and $92 \%$ to $99 \%$ respectively [4,15]. In our study, out of 21 positive exams, 8 exams were confirmed to be true positives and 4 were ruled to be false negatives. Unfortunately, there were 9 studies that were neither confirmed nor ruled out and are therefore of unknown significance. This appears to have occurred when there was no need or no value to intervening in those patients. Either the condition stabilized or improved, or the patient was discharged to palliative care or hemodialysis for complete kidney failure. There were 2 negative exams with known RAS (false negatives) and 267 negative

Table 4. Prevalence of specific co-morbidities and characteristics by RAS positive and negative Doppler exams.

\begin{tabular}{|c|c|c|c|c|c|}
\hline Variable & $\begin{array}{l}(+) \text { Doppler for } \\
\text { RAS }(n=21)\end{array}$ & (-) Doppler for RAS (n=270) & Odds Ratio & $95 \% \mathrm{CI}$ & Pvalue \\
\hline Age* & $65.1 \pm 15.5$ & $59.9 \pm 15.6$ & - & - & 0.145 \\
\hline Age; younger or older than $50^{*}$ & & & 2.27 & $0.65,7.92$ & 0.189 \\
\hline$<50$ & $3(14.3 \%)$ & $74(27.4 \%)$ & $(>50 /<50)$ & - & - \\
\hline$>50$ & $18(85.7 \%)$ & $196(72.6 \%)$ & - & - & - \\
\hline Gender & & & 1.6 & $0.63,4.09$ & 0.323 \\
\hline Male & $14(66.7 \%)$ & $150(55.6 \%)$ & (male/female) & - & - \\
\hline Female & $7(33.3 \%)$ & $120(44.4 \%)$ & - & - & - \\
\hline Hypertension & $20(95.2 \%)$ & $193(71.5 \%)$ & 7.98 & $1.05,60.5$ & 0.018 \\
\hline Chronic kidney disease & $10(47.6 \%)$ & $78(28.9 \%)$ & 2.24 & $0.91,5.48$ & 0.072 \\
\hline Diabetes mellitus & $6(28.6 \%)$ & $100(37.0 \%)$ & 0.68 & $0.26,1.81$ & 0.437 \\
\hline Cardiovscular disease & $10(47.6 \%)$ & $113(41.9 \%)$ & 1.26 & $0.52,3.08$ & 0.606 \\
\hline Acute kidney injury* & $16(76.2 \%)$ & $228(84.4 \%)$ & 0.59 & $0.21,1.70$ & 0.353 \\
\hline $\begin{array}{l}\text { Uncontrolled hypertension/ } \\
\text { flash pulmonary edema* }\end{array}$ & $11(52.4 \%)$ & $61(22.6 \%)$ & 3.78 & $1.53,9.29$ & 0.002 \\
\hline Serum creatinine $(\mathrm{mg} / \mathrm{dL})^{*}$ & $3.8 \pm 4.5$ & $3.5 \pm 3.5$ & - & - & 0.757 \\
\hline Blood urea nitrogen $(\mathrm{mg} / \mathrm{dL})^{*}$ & $51.6 \pm 45.0$ & $53.7 \pm 37.9$ & - & - & 0.829 \\
\hline BUN to creatinine ratio* & $17.3 \pm 9.5$ & $18.4 \pm 10.8$ & - & - & 0.633 \\
\hline Kidney Length (cm) & $11.3 \pm 1.7$ & $11.6 \pm 1.7$ & - & - & 0.374 \\
\hline Resistive index & $0.74 \pm .13$ & $0.81 \pm .09$ & - & - & 0.016 \\
\hline
\end{tabular}


exams with no known RAS. Without appropriate confirmatory testing on these patients, there is no way to determine the true number of true and false negative exams. This problem, and the small sample size of the study (excluding the 9 studies of unknown significance), contribute to the limited applicability of the sensitivity and specificity calculations. Regardless of this, the sensitivity and specificity of RHDUS was comparable to that reported for RHDUS in other studies $[4,15]$. Sensitivity and specificity were $80 \%$ and $98.5 \%$ respectively with a positive predictive value of $66.7 \%$ and negative predictive value of $99.3 \%$. This may suggest that portable RHDUS in the ICU has comparable sensitivity and specificity to non-portable Doppler using both direct and indirect parameters. Our numbers may also be inflated due to unknown number false negatives among atherosclerotic patients and false positives among hemodynamically unstable patients that were not confirmed by direct methods (i.e., angiography, MRA, CTA, non-portable RDUS).

Another limitation of portable Doppler's intrarenal measurements can be seen in the discrepancy of bilateral vs unilateral disease seen on Doppler. Out of 7 exams with bilateral evidence of RAS, 3 exams were ruled out and 3 were not followed. A single study was confirmed for bilateral RAS by renal angiography and this patient had a previously known history of RAS. Out of 14 exams showing unilateral evidence of RAS, only one was ruled out by other testing and was found to have severe hydronephrosis and a high-grade obstruction. This discrepancy in false positives among bilateral and unilateral abnormalities can be explained by the inability of intrarenal parameters to distinguish stenosis from causes of systemic hemodynamic instability or aortopathies.

A RI below 0.55 is suggestive of RAS; but, in this study the mean RI for positive RAS on Doppler was well elevated above even normal limits (0.74) though it was significantly lower than RAS negative patients (0.81), $\mathrm{p}=.016$. Only one kidney evaluated and found to have other abnormalities suggestive of RAS had an RI below 0.55 . The 2 lowest values for RI were 0.45 and 0.55 . This is likely due to high prevalence of microvascular or parenchymal renal disease associated with high prevalence of hypertension and diabetes, and advanced age. These findings agree with similar findings in other studies [14].

The association between RAS and increased age, hypertension, and atherosclerotic disease has already been established by multiple studies [13,14,16-19]. Univariate analysis in this study showed a significant association between previously documented hypertension and positive RDHUS for RAS ( $\mathrm{p}=.018$ ) and even stronger significance between hypertensive urgency or flash pulmonary edema on admission and positive RHDUS for RAS ( $\mathrm{p}=.002)$. Examination for renal artery stenosis is indicated by resistant or poorly controlled blood pressure, coexisting atherosclerosis, kidney injury of unknown etiology, and atrophic or asymmetric kidneys [1,7]. This study confirms that poorly controlled hypertension or hypertensive urgency and/or flash pulmonary edema is a good indicator for a Doppler evaluation for RAS. Chronic kidney disease was seen in $47.6 \%$ of RAS-positive exams vs $28.9 \%$ in RAS-negative exams $(\mathrm{p}=.072)$ and there was a 5.2 year difference between the mean ages of RAS-positive and RAS-negative exams; 65.1 and 59.9 respectively $(\mathrm{p}=.145)$. Increasing the patient pool may be indicated for future studies to allow for multivariate analysis and reveal more significance among known predictors of RAS.

It has also been shown that hypertension is more likely secondary to RAS in patients that develop it over 55 (atherosclerosis) and under 30 (fibromuscular dysplasia) and the development of hypertension between the age of 30 and 55 is most likely essential hypertension [20]. No patients younger than 39 were RAS-positive on RHDUS. Only 3 patients under 30 years old had documented hypertension and none had fibromuscular dysplasia. All three were negative for RAS on Doppler. Our study suggests that the likelihood of RAS-positive RHDUS in patients younger than 50 without other predisposing factors such as uncontrolled hypertension, atherosclerotic disease or fibromuscular dysplasia is very small; though age as a variable could not reach statistical significance in our small sample.

Out of all the RAS-positive studies, only 5 had AKI without other documented diabetes or cardiovascular disease. Of these 5 , the youngest was 65 years and they all had documented hypertension. Our study also showed that the prevalence of acute kidney injury was lower in RAS-positive exams compared to RAS-negative exams without any significant difference. This may be evidence that AKI alone is a poor predictor of RAS and not an appropriate indication for RAS screening without other risk factors present. The renal insufficiency in these patients was likely due to intrinsic causes as indicated by the $88 \%$ prevalence of microvascular or parenchymal renal disease among our studied patients.

Together with grayscale sonography, Doppler is used in the ICU to assess for causes of renal failure. The question to be answered by the ultrasound was commonly "please rule out obstruction or stenosis." Keyserling, et al. [20] showed that the use of renal sonography in the assessment of acute and acute-on-chronic kidney failure for obstructive uropathy in patients that are not predisposed to obstruction is not indicated in the ICU setting; as obstructive uropathy is a rare finding in these circumstances. If RHDUS is not indicated for the assessment of RAS in any subgroup of ICU patients then the use of portable RHDUS in the ICU in the assessment of renal failure is not indicated in those patients. This could potentially save healthcare dollars for the patients and the institution. While the cost of Doppler ultrasound varies widely based on regional and institutional differences and on insurance status, the cost savings could be significant. At our institution, charges for a Doppler ultrasound evaluation of the kidneys (CPT code 93975) are $\$ 437$ for the physician fee and $\$ 1,033$ for the hospital fee.

\section{Conclusion}

RHDUS is comparable to non-portable Doppler in sensitivity and specificity when screening for RAS and can be an effective tool in the evaluation of patients with risk factors (i.e., smoking, hypertension, diabetes, fibromuscular dysplasia) and/or suspected RAS. However, the limitation of RHDUS may not make a preferred screening tool in certain populations of critically-ill patients, especially patients with hemodynamic instability or major vasculopathies that effect flow to the kidneys. ICU patients presenting with AKI are rarely positive for RAS by RHDUS screening unless they have a high suspicion for RAS or also presenting with hypertensive crisis or urgency

In this study, we hypothesized that the number of managementaltering positive RHDUS studies would be very low, less than $5 \%$. Out of 291 studies we saw that just $21(7 \%)$ were positive, $8(3 \%)$ were confirmed to have RAS, and only $3(1 \%)$ of those underwent intervention. RHDUS is a useful screening test in patients with a high suspicion of RAS by risk factors (or with a history of RAS) and presenting with a hypertensive crisis or resistant hypertension. In many ICU patients such as those under 50, presenting only with AKI, or with hemodynamic instability the use of RHDUS screening for RAS is very low yield and shouldn't be used. 


\section{References}

1. Olin JW (2002) Atherosclerotic renal artery disease. Cardiol Clin 20: 547-562. [Crossref]

2. Radermacher J, Brunkhorst R (1998) Diagnosis and treatment of renovascular stenosis-a cost-benefit analysis. Nephrol Dial Transplant 13: 2761-2767.[Crossref]

3. Safian RD, Textor SC (2001) Renal-artery stenosis. $N$ Engl J Med 344: 431-442. [Crossref]

4. Piecha G, Wiecek A, Januszewicz A (2012) Epidemiology and optimal management in patients with renal artery stenosis. J Nephrol 25: 872-878.[Crossref]

5. House MK, Dowling RJ, King P, Gibson RN (1999) Using dopplersonography to reveal renal artery stenosis: An evaluation of optimal imaging parameters. AJR Am J Roentgenol 173: 761-765.[Crossref]

6. Olin JW, Piedmonte MR, Young JR, DeAnna S, Grubb M, et al. (1995) The utility of duplex ultrasound scanning of the renal arteries for diagnosing significant renal artery stenosis. Ann Intern Med 122: 833-838.[Crossref]

7. Solar M, Zizka J, Krajina A, Michl A, Raupach J, et al. (2011) Comparison of duplex ultrasonography and magnetic resonance imaging in the detection of significant renal artery stenosis. Acta Medica (Hradec Kralove) 54: 9-12.[Crossref]

8. Miralles M, Cairols M, Cotillas J, Gimenez A, Santiso A (1996) Value of doppler parameters in the diagnosis of renal artery stenosis. J Vasc Surg 23: 428-435.[Crossref]

9. Hansen KJ, Tribble RW, Reavis SW, Canzanello VJ, Craven TE, et al. (1990) Renal duplex sonography: evaluation of clinical utility. J Vasc Surg 12: 227-236.[Crossref]

10. AbuRahma AF, Srivastava M, Mousa AY, Dearing DD, Hass SM, et al. (2012) Critical analysis of renal duplex ultrasound parameters in detecting significant renal artery stenosis. J Vasc Surg 56: 1052-1059, 1060-e1.[Crossref]
11. Case J, Khan S, Khalid R, Khan A (2013) Epidemiology of acute kidney injury in the intensive care unit. Crit Care Res Pract: 479730.[Crossref]

12. Shukla AN, Madan TH, Jayaram AA, Kute VB, Rawal JR, et al. (2013) Prevalence and predictors of renal artery stenosis in patients undergoing peripheral and coronary angiography. Int Urol Nephrol 45: 1629-1635.[Crossref]

13. Hansen KJ, Edwards MS, Craven TE, Cherr GS, Jackson SA, et al. (2002) Prevalence of renovascular disease in the elderly: a population-based study. J Vasc Surg 36: 443451.[Crossref]

14. Ripollés T, Aliaga R, Morote V, Lonjedo E, Delgado F, et al. (2001) Utility of intrarena doppler ultrasound in the diagnosis of renal artery stenosis. Eur J Radiol 40: 54-63. [Crossref]

15. Nazzal MM, Hoballah JJ, Miller EV, Sharp WJ, Kresowik TF, et al. (1997) Renal hilar doppler analysis is of value in the management of patients with renovascular disease. Am J Surg 174: 164-168.[Crossref]

16. Olin JW, Melia M, Young JR, Graor RA, Risius B (1990) Prevalence of atherosclerotic renal artery stenosis in patients with atherosclerosis elsewhere. Am J Med 88: 46N-51N. [Crossref]

17. Kohler TR, Zierler RE, Martin RL, Nicholls SC, Bergelin RO, et al. (1986)Noninvasive diagnosis of renal artery stenosis by ultrasonic duplex scanning. J Vasc Surg 4: 450456.[Crossref]

18. Leertouwer TC, Pattynama PM, van den Berg-Huysmans A (2001) Incidental rena artery stenosis in peripheral vascular disease: A case for treatment? Kidney Int 59: 1480-1483.[Crossref]

19. Schwartz CJ, White TA (1964) Stenosis of renal artery: An unselected necropsy study. Br Med J 2: 1415-1421.[Crossref]

20. Keyserling HF, Fielding JR, Mittelstaedt CA (2002) Renalsonography in the intensive care unit: when is it necessary? J Ultrasound Med 21: 517-520.[Crossref]

Copyright: (C2017 Amarneh M. This is an open-access article distributed under the terms of the Creative Commons Attribution License, which permits unrestricted use, distribution, and reproduction in any medium, provided the original author and source are credited. 